\title{
THE TATE CONJECTURE FOR $t$-MOTIVES
}

\author{
YUICHIRO TAGUCHI
}

(Communicated by Eric Friedlander)

\begin{abstract}
A version of the Tate conjecture is proved for $\varphi$-modules of " $t$ motive type".
\end{abstract}

In this note, we formulate a version of the Tate conjecture for $\varphi$-modules, and give a proof of it in a special but essential case. Similar results have been obtained independently by Tamagawa [3] in a more general setting.

Let $K$ be an algebraic function field in one variable over a finite field, whose field of constants is $\mathbb{F}_{q}, \pi$ a place of $K$, and $K_{\pi}$ the completion of $K$ at $\pi$. Let $k$ be any field containing $\mathbb{F}_{q}$. We set $K_{k}:=k \otimes_{\mathbb{F}_{q}} K$, and denote by $K_{k, \pi}$ the completion of $K_{k}$ with respect to the $\pi$-adic topology (these may not be fields). Let $\sigma$ be the endomorphism ( $q$-th power Frobenius of $k) \otimes\left(\mathrm{id}_{K}\right)$ of $K_{k}$, and also its natural extension to $K_{k, \pi}$. By a $\varphi$-module $(M, \varphi)$ (or simply $M$ ) over $K_{k}$ (resp. over $K_{k, \pi}$ ), we mean a free $K_{k}$-module (resp. $K_{k, \pi}$-module) $M$ of finite rank equipped with a $\sigma$-semi-linear map $\varphi: M \rightarrow$ $M$. Morphisms of $\varphi$-modules are defined naturally. Tensor products $M \otimes N$ (with diagonal $\varphi$-action) exist. Internal homs $\operatorname{Hom}(M, N)$ (with $\varphi: " f \mapsto$ $\varphi_{N} \circ f \circ \varphi_{M}^{-1}$ ") may or may not exist. For a $\varphi$-module $M$, let $M^{\varphi}$ denote the fixed part of $M$ by $\varphi$. This is a $K$-subspace (resp. $K_{\pi}$-subspace) of $M$. If the internal hom $H=\operatorname{Hom}(M, N)$ exists, then $H^{\varphi}$ is the space $\operatorname{Hom}_{\varphi}(M, N)$ of $\varphi$-module homomorphisms of $M$ to $N$. Now the Tate conjecture in our context is

Conjecture. Let $M$ be a $\varphi$-module over $K_{k}$. If $k$ is of finite type over $\mathbb{F}_{q}$, then the natural map of $K_{\pi}$-vector spaces

$$
K_{\pi} \otimes_{K} M^{\varphi} \rightarrow\left(K_{k, \pi} \otimes_{K_{k}} M\right)^{\varphi}
$$

is an isomorphism.

In general, this is not true (cf. [3]).

Equivalently, by fixing a $K_{k}$-basis of $M$, the conjecture can be stated also as follows: Let $A$ be a matrix in $\mathrm{M}_{r}\left(K_{k}\right)$. Consider the linear Frobenius equation

$$
A X^{\sigma}=X \text {, }
$$

Received by the editors April 28, 1994.

1991 Mathematics Subject Classification. Primary 11G09.

Partially supported by JSPS Postdoctoral Fellowships for Research Abroad.

(C)1995 American Mathematical Society 
where the indeterminate $X$ is considered in $K_{k, \pi}^{\oplus r}$, on which $\sigma$ acts componentwise. Let $V$ (resp. $V_{\pi}$ ) be the space of solutions of $\left(^{*}\right)$ in $K_{k}^{\oplus r}$ (resp. $K_{k, \pi}^{\oplus r}$ ). Then the natural map of $K_{\pi}$-vector spaces $K_{\pi} \otimes_{K} V \rightarrow V_{\pi}$ is an isomorphism.

These problems can (and should) be considered also with $K_{k}$ replaced by certain localizations of it.

The injectivity is easy to see, so the essence is in the surjectivity.

A reduction can be made: suppose $K^{\prime}$ is a subfield of $K$ which contains $\mathbb{F}_{q}$ and over which $K$ is finite. Let $\pi^{\prime}$ be the restriction of $\pi$ to $K^{\prime}$. A $\varphi$-module $M$ over $K_{k}$ can be regarded as a $\varphi$-module over $K_{k}^{\prime}$. If the conjecture is true for $\left(K^{\prime}, \pi^{\prime}, M\right)$, then it is also true for $(K, \pi, M)$ (use the identification $K_{\pi^{\prime}}^{\prime} \otimes_{K^{\prime}} K=\prod_{\pi \mid \pi^{\prime}} K_{\pi}$, etc.). So we may and do assume $K=\mathbb{F}_{q}(t)$ and identify $\pi$ with a monic irreducible element of $\mathbb{F}_{q}[t]$. Replacing $K$ again by the subfield $\mathbb{F}_{q}(\pi)$, we may assume $\pi=t$ (so $K_{k}$ is the polynomial ring $k[t]$ to which the inverses of all monic polynomials in $\mathbb{F}_{q}[t]$ have been adjoined, and $K_{k, \pi}=$ $k((t)))$.

Now we prove the conjecture assuming that $k$ is a function field in one variable over $\mathbb{F}_{q}$ (this is not essential) and that $M$ comes from $t$-motives of characteristic different from $\pi$, by which we mean the following (cf. [1]): there exist a non-zero element $\theta$ of $k$ and positive integers $d$ and $d^{\prime}$ such that the map $\varphi$ is represented with respect to some $K_{k}$-basis of $M$ by a matrix $A$ of the form

$$
A=(t-\theta)^{d} B^{-1},
$$

where $B$ is a matrix in $\mathrm{M}_{r}(k[t])$ with $\operatorname{det} B$ of the form $u(t-\theta)^{d^{\prime}}, u \in k^{\times}$ (so we allow any $A \in \mathrm{GL}_{r}\left(k\left[t, \frac{1}{t-\theta}\right]\right)$ in $\left(^{*}\right)$, which may not be in $\left.\mathbf{M}_{r}\left(K_{k}\right)\right)$.

We will show that, if the equation $\left(^{*}\right)$ has a solution $\underline{\hat{x}}$ in $K_{k, \pi}^{\oplus r}$, then it has a solution $\underline{x}$ in $K_{k}^{\oplus r}$ which is sufficiently close (in the $t$-adic $=\pi$-adic topology) to $\underline{\hat{x}}$ (so that, if $\left(\underline{\hat{x}}_{i}\right)$ is a basis for $V_{\pi}$, so is $\left(\underline{x}_{i}\right)$ for $V$ ). By assumption, we have

$$
(t-\theta)^{d} \underline{\hat{x}}^{\sigma}=B \underline{\hat{x}}, \quad \theta \neq 0 .
$$

Write $(t-\theta)^{d}=\sum_{i=0}^{d} \theta_{i} t^{i} \quad$ (with $\left.\theta_{i} \in k\right) ; B=\sum_{i=0}^{N} B_{i} t^{i} \quad$ (with $B_{i} \in \mathbf{M}_{r}(k)$ ); and $\underline{\hat{x}}=\sum_{i \geq 0} x_{i} t^{i} \quad\left(\right.$ with $\left.x_{i} \in k^{\oplus r}\right)$. Then (**) yields

$$
\theta_{0} x_{i}^{\sigma}+\cdots+\theta_{d} x_{i-d}^{\sigma}=B_{0} x_{i}+\cdots+B_{N} x_{i-N}, \quad i \geq 0 .
$$

(Here negatively indexed terms are zero.) For any valuation $v$ of $k$, let $v\left(x_{i}\right)$ denote the minimum of the valuations of the entries of $x_{i}$, and $v(B)$ the minimum of the valuations of the entries of $B_{i}$ for all $i \geq 0$. If $v(\theta) \leq 0$, then

$$
v\left(x_{i}\right) \geq \min \left\{v\left(x_{i-1}\right), \cdots, v\left(x_{i-d}\right), \frac{v\left(x_{i}\right)+v(B)}{q}, \cdots, \frac{v\left(x_{i-N}\right)+v(B)}{q}\right\},
$$

so we see recursively that $v\left(x_{i}\right) \geq v(B) /(q-1)$ for all $i \geq 0$. If $v(\theta)>$ 0 , we replace $X$ in $\left(^{* *}\right)$ by $\theta^{-e} X$ (resp. $B$ by $\left.\theta^{e(q-1)} B\right)$ for some $e$ to have $v\left(\theta^{e(q-1)} B\right) \geq 0$. Then Anderson's arguments ${ }^{1}$ in $\S 4$ of [2] imply the

\footnotetext{
${ }^{1}$ His arguments there show in particular the following: let $\theta$ be the integer ring of the completion $k_{v}$ of $k$ at the place $v$. Let $B$ be a matrix in $\mathrm{M}_{r}(\mathscr{O} \llbracket t \rrbracket)$ such that $\operatorname{det} B=u(t-\theta)^{d^{\prime}}$ with a non-zero $u \in \mathscr{O}$. Then any solution $X$ to the equation $(t-\theta)^{d} X^{\sigma}=B X$ in $k_{v} \llbracket t \rrbracket^{\oplus r}$ is
} 
holomorphy of the new solution $\theta^{e} \underline{\hat{x}}$, hence the old solution $\underline{\hat{x}}$ satisfies $v\left(x_{i}\right) \geq$ $-e v(\theta)$ for all $i \geq 0$. Thus the values $v\left(x_{i}\right), i \geq 0$, are bounded below for all valuations $v$ of $\bar{k}$, by constants which are non-negative for almost all $v$. By the bounded height theorem, there appear in fact only finitely many $x_{i}$ 's in the sequence $x_{0}, x_{1}, \cdots$. Accordingly, there appear only finitely many equations $\left({ }^{* *}\right)$, and one can choose a "periodic" (except for finitely many terms) solution $\underline{x}=\sum x_{i}^{\prime} t^{i}$ in $K_{k, \pi}^{\oplus r}$ to $\left.{ }^{* * *}\right)$ closely enough to the original $\underline{\hat{x}}$. Periodicity implies that $\underline{x}$ is rational with denominator in $\mathbb{F}_{q}[t]$, hence $\underline{x} \in K_{k}^{\oplus r}$.

\section{ACKNOWLEDGMENT}

I thank Greg Anderson for a careful reading of the manuscript and helpful comments. I also thank Akio Tamagawa for his encouragement, and the Institute for Advanced Study for its hospitality.

\section{REFERENCES}

1. G. Anderson, t-motives, Duke Math. J. 53 (1986), 457-502.

2. __ On Tate modules of formal t-modules, Internat. Math. Res. Notices 2 (1993), 41-52.

3. A. Tamagawa, The Tate conjecture for A-premotives, preprint.

Tokyo Metropolitan University, Hachioji, Tokyo 192-03, JaPan

E-mail address: taguchi@math.metro-u.ac.jp

automatically in $\mathscr{O} \llbracket t \rrbracket^{\oplus r}$. (The proof is found in the last page of [2], except that we need to use his Lemma 7 for a more general $\Phi=B$ as above, with the maximal ideal $\mathscr{M}^{\text {sep }}$ in the statement replaced by the integer ring $\mathscr{O}$ sep of a separable closure of $k_{v}$. This can be proved easily by looking at each term of the $t$-adic expansion of the given equation, just as we did in $(* * *)$.) 